\title{
Identification and analysis of the promoter region of the STGC3 gene
}

\author{
Suyun Li, Lili Wang, Xiusheng He, Yuanjie Xie, Zhiwei Zhang
}

Cancer Research Institute of the University of South China, Hengyang, China

Submitted: 20 November 2012

Accepted: 21 March 2013

Arch Med Sci 2015; 11, 5: 1095-1100

DOI: 10.5114/aoms.2015.49213

Copyright $\odot 2015$ Termedia \& Banach

\section{Abstract}

Introduction: Nasopharyngeal carcinoma (NPC) is a common malignant tumor of the head and neck. The STGC3 gene is related to development of nasopharyngeal cancer. The aim of this study is to explore the promoter region of the STGC3 gene.

Material and methods: The bioinformatic technique was applied to predict its promoter region and construct the gene promoter region luciferase for the gene vector and transfection of the human embryonic kidney epithelial 293T cell line, human nasopharyngeal carcinoma CNE2 cell line and immortalized nasopharyngeal epithelial NP69 cell line. The recombinant plasmid pGL3-en283, pGL3-en281, pGL3-en571, empty plasmid pGL3-control, negative control pGL3-enhance and internal control of marine intestine luciferase expression vector pRL-SV40 were transfected into NP69 cells, 293T cells and CNE2 cells. Dual luciferase activity detection showed luciferase luminescence values and marine intestine luciferase luminescence values. Relative luciferase activity (RLA) in each cell was calculated.

Results: We observed strong promoter activity of plasmid pGL3-en283, pGL3en281 and pGL3-en571 in NP69, 293T and CNE2 cells compared with the negative control pGL3-enhance plasmid. Among them, pGL3-en281 showed the strongest promoter activity, and these three kinds of recombinant plasmids showed stronger promoter activity in 293 T cells than in CNE2 cells.

Conclusions: The pGL3-en281 plasmid showed stronger promoter activity than pGL3-en571 in the three cells, indicating that -11048 bp to $-653 \mathrm{bp}$ might be the core promoter region.

Key words: nasopharyngeal carcinoma, STGC3, promoter, bioinformatics.

\section{Introduction}

Nasopharyngeal carcinoma (NPC) is a common malignant tumor in the head and neck that is prevalent in many provinces in southern China and Southeast Asia, with significant ethnic and regional distribution [1]. The occurrence of nasopharyngeal carcinoma is a complex process that involves multiple factors, multiple stages, and multiple steps [2, 3]. It is associated with Epstein-Barr virus infection, environment, diet and genetic factors, which involve many oncogenes and/or changes in the tumor suppressor genes [4,5]. The inactivation of cancer suppressor genes plays an important role [6].

STGC3 is a potential tumor suppressor related to NPC [7-9]. The expression of STGC3 was decreased in the NPC cell lines and the NPC tissues $[8,9]$. Restoring the expression of STGC3 in the tumor cell line CNE2

\author{
Corresponding author: \\ Xiusheng $\mathrm{He}$ \\ Cancer Research Institute \\ of the University \\ of South China \\ Hengyang, Hunan Province \\ 421001, China \\ Phone: +86 13975455786 \\ Fax: +86 7348282207 \\ E-mail: \\ hexiusheng118@163.com
}


significantly inhibits growth and proliferation of the cells in vitro and in vivo $[10,11]$. In our study, the STGC3 gene was cloned from a nasopharyngeal cancer related gene (GenBank accession number AY078383) in expressed sequence tags (ESTs) at the $3 p 21$ region of the nasopharyngeal high-frequency loss of heterozygosity $(\mathrm{LOH})$ site through a gene positional candidate cloning strategy. The preliminary findings showed a significant difference between the STGC3 gene in normal and cancer nasopharyngeal tissues for its inhibition of tumor cell growth and proliferation [7-11]. It was found that the LG domain is necessary for the cancer inhibition of STGC3 genes [12-15].

So far, the transcriptional regulation of the STGC3 gene is less known. To reveal the expression and regulation of the STGC3 gene and clarify the down-regulation mechanisms of nasopharyngeal cancer cells, the STGC3 promoter region was analyzed through bioinformatics [16-18] in this study. It was preliminarily analyzed by construction of a reporter gene vector and transfection techniques. The gene regulation and expression were further explored.

\section{Material and methods}

\section{Cells and vectors}

The human embryonic kidney epithelial 293T cell line was provided by the Institute of Cancer Research, Xiangya Medical College, Central South University. The human nasopharyngeal carcinoma CNE2 cell line and immortalized nasopharyngeal epithelial cell line NP69 were persevered in our institute. The 293T line was persevered in 10\% fetal bovine serum medium of high glucose DMEM, the CNE2 line in RPMI 1640 medium with 10\% newborn calf serum, and NP69 in keratinocyte serum-free medium at $37^{\circ} \mathrm{C}$ with $5 \% \mathrm{CO}_{2}$ and saturated humidity. pGEM T-Easy vector, pGL3 control vector, pGL3-enhance vector, and pRL-SV40 Vector were purchased from Promega Corporation. The JM109 strain was provided by the Institute of Cancer Research, Xiangya Medical College.

\section{Reagent}

Calf serum was purchased from the Evergreen Company (Hangzhou, China). High glucose medium DMEM and 1640 medium were purchased from Hyclone. K-SFM culture medium without serum was purchased from Invitrogen. The plasmid extraction kit and DNA Marker DL2000 were purchased from Takara. Restriction endonuclease (Mlul and BgllI) and T4 DNA ligase were purchased from NEB. The gel extraction kit was purchased from Shanghai Huashun Biological Company. Dual-Luciferase \& Reporter Assay System was purchased from Promega. Lipofectamine 2000 liposome transfection reagents were purchased from Invitrogen. Yeast powder and tryptone were purchased from Oxoid. Pfu DNA Polymerase was purchased from Fermentas.

\section{Bioinformatics analysis of the regulatory region at 5' end of STGC3 gene}

The first base of initiation codon ATG starting translating from the STGC3 gene was marked +1 . STGC3 gene sequence blast was carried using NCBI database, and a total of 6271 bp sequences namely the upstream 5000 bp sequences and STGC3 gene sequences were found out. Analysis of this $6271 \mathrm{bp}$ sequence by UCSC/Ensembl showed that the promoter active region was in chr3: 49,295,000-49, 298,000, where it was marked as a promoter, transcription initiation site and $\mathrm{CpG}$ island analysis sequence.

Online software NNPP (http://www.fruitfly.org/ seq_tools/promoter.html) and Promoter 2.0 (http:// www.cbs.dtu.dk/services/Promoter/) were applied for prediction of candidate STGC3 gene promoter sequence and transcription initiation sites. MethPrimer (http://www.urogene.org/methprimer/) and Cpgplot (http://www.ebi.ac.uk/Tools/emboss/cpgplot/) were applied for analysis of the CpG island in the STGC3 gene.

\section{Construction of the reporter gene vector in the STGC3 gene promoter region}

Normal human whole blood gDNA was extracted by the Promega genomic DNA extraction kit. Primer 5.0 was applied for the 5 end primers in the regulatory region of STGC3. Mlul restriction sites (underlined) and protective bases were introduced to the 5' end of the forward primer, and Bglll restriction sites (underlined) and protective bases were introduced to the 5' end of the reverse primer. Its primer sequences were: sense: cgacgcgtTTAGCTGGACATGGTGGCGT, antisense: gaagatctATCTCTGTCCCAAAGTGACA, sense: cgacgcgtCCTGAGCGACAGAGTGAG, antisense: gaagatctCGAGTAGCTGGGACAACA, sense: cgacgcgtCCTGAGCGACAGAGTGAG, antisense: gaagatctCGAGTAGCTGGGACAACA.

The size of amplified fragments was $283 \mathrm{bp}$ (-1360 to -1077$), 281$ bp (-934 bp to $-653 \mathrm{bp})$, 571 bp ( -500 bp to +72 bp) respectively. High-fidelity DNA polymerase Pfu DNA Polymerase was applied for PCR amplification of the three fragments. The amplified product added a poly (A) tail, the amplified product was introduced to the pGEM-T vector. After confirmed by sequencing, the fragments were digested with Mlul and BgllI and connected to the pGL3-Enhancer linearized vector prepared by Mlul and Bglll double digestion, regulatory sequences in the $5^{\prime}$ end of the 
STGC3 gene and the recombinant plasmid of the firefly luciferase reporter gene were built. The recombinant plasmid was tested and sequenced, and the positive cloned plasmid was used for cell transfection.

\section{Activity detection of gene promoter region} in the recombinant plasmid STGC3

NP69 cells, 293T cells and CNE2 cells with relatively high expression of the STGC3 gene were chosen as target cells for the follow-up study. These three cells were vaccinated in a 24-well culture plate, and when these cells had grown to 80$90 \%, 800$ ng of pGL3 recombinant plasmid and 10 ng of phRL-SV40 were transfected according to the instructions of Lipofectamine 2000 liposomes. While establishing pGL3-Enhancer as a negative control and pGL3-Control as a positive control for transfection of $30 \mathrm{~h}$, luciferase reporter gene activity was detected following dual luciferase assay kit steps. The culture solution was discarded and the cells were washed with cold PBS twice. Then $100 \mu$ of passive lysis buffer (PLB) was added to each well, and it was placed on a shaker at room temperature for slow shaking for 15-20 min. After repeated freezing and thawing, cell lysates were transferred to a $0.5 \mathrm{ml}$ centrifuge tube for brief centrifugation of $10 \mathrm{~s}$. Then $20 \mu \mathrm{l}$ of cell lysate was added to $100 \mu \mathrm{l}$ of fluorescence luciferase substrate (LAR II), and liquid scintillation luciferase was measured for the luminescence value after mixing. Then $100 \mu$ l of reaction terminated liquid (Stop \& Glo Reagent) was added and mixed for measurement of internal standard Renilla luciferase with a liquid scintillation counter. The ratio of the two is the relative luciferase activity (RLA). Each recombinant plasmid and control plasmid was transfected and detected three times.

\section{Results}

\section{Bioinformatics analysis of 5' regulatory region in STGC3 gene}

Based on the NCBI database, sequence alignment was applied for the STGC3 gene to identify the full sequence of the STGC3 gene and its 5000 bp upstream sequence for promoter prediction. Based on UCSC/Ensembl, the promoter, intron and exon sequences were identified and the promoter active region was chr3: 49,295,000 bp-49, $298,000 \mathrm{bp}$. This $3000 \mathrm{bp}$ sequence was extracted and promoter analysis was applied as the first base $A$ in translation initiation codon ATG of the STGC3 gene marked as +1 . It showed that there were many promoter regions in the STGC3 gene. Among them six regions scored at least 0.8 , and the highest score was 1.0 in the -845 bp and -795 bp region (the higher the score was, the stronger the promoter activity).

Promoter 2.0 software prediction showed that this gene had two transcription start site (TSS), one was at site -2348 bp and the other was at site $-948 \mathrm{bp}$. The MethPrimer online program analysis showed two $\mathrm{CpG}$ islands: the first $\mathrm{CpG}$ island was located at $-1805 \mathrm{bp}$ to $-1705 \mathrm{bp}$ with length of $101 \mathrm{bp}$, and the other CpG island was located at $-900 \mathrm{bp}$ to $-684 \mathrm{bp}$ with length of 217 bp. Cpgplot software analysis showed one CpG island at $-900 \mathrm{bp}$ to $-684 \mathrm{bp}$ with length of 217 bp. Homology analysis in comparative genomics of STGC3 genes in other vertebrates found that the STGC3 gene in humans was highly homologous to that in chimpanzees, monkeys, dogs, and other animals, especially the exon region (blue area), which indicates that the STGC3 gene is a relatively conservative gene (Figure 1 ).

\section{Reporter gene vector construction and activity detection of promoter region in STGC3}

Based on the bioinformatics results, a figure of the possible STGC3 gene promoter region from -3046 bp to -46 bp was drawn. The grey region indicated that the promoter region had a score of 0.8 or more, with two gene transcription initiation sites, one at -948 bp upstream of the start codon, the other at upstream -2348 bp. CpG island 1 was located at -1805 bp to -1705 bp with length of $101 \mathrm{bp}$, and a CpG island was located at $-900 \mathrm{bp}$ to $-684 \mathrm{bp}$ with length of $217 \mathrm{bp}$ (Figure $2 \mathrm{~A}$ ). Based on the bioinformatics results and promoter region, the vectors were constructed. The $-1360 \mathrm{bp}$ to -1048 bp region contained a fragment with a prediction score of 0.91 and GC box. The -1048 bp to -653 bp region contained a fragment with a prediction score of 1.0 , transcription initiation sites, CPG island and GC boxes. The -541 bp to +72 bp region contained a fragment with a prediction score of 0.97 and CAAT box (Figure 2 B). Human blood DNA was the template and a high-fidelity enzyme was used for PCR amplification of the target fragment. The recombinant PGL3-enhance carrier was double digested by restriction enzymes Mlul and Bglll and sequenced, showing that the inserted sequence in the recombinant plasmid and NCBI was coherent (Figure $2 \mathrm{C}$ ).

The recombinant plasmid pGL3-en283, pGL3en281, pGL3-en571 empty plasmid pGL3-control, pGL3-enhance and internal control of marine intestine luciferase expression vector pRL-SV40 were transfected into NP69 cells, 293T cells and CNE2 cells. The dual luciferase activity detection kit showed the luciferase luminescence value and marine intestine luciferase luminescence value. The RLA in each cell was calculated (Table I, 


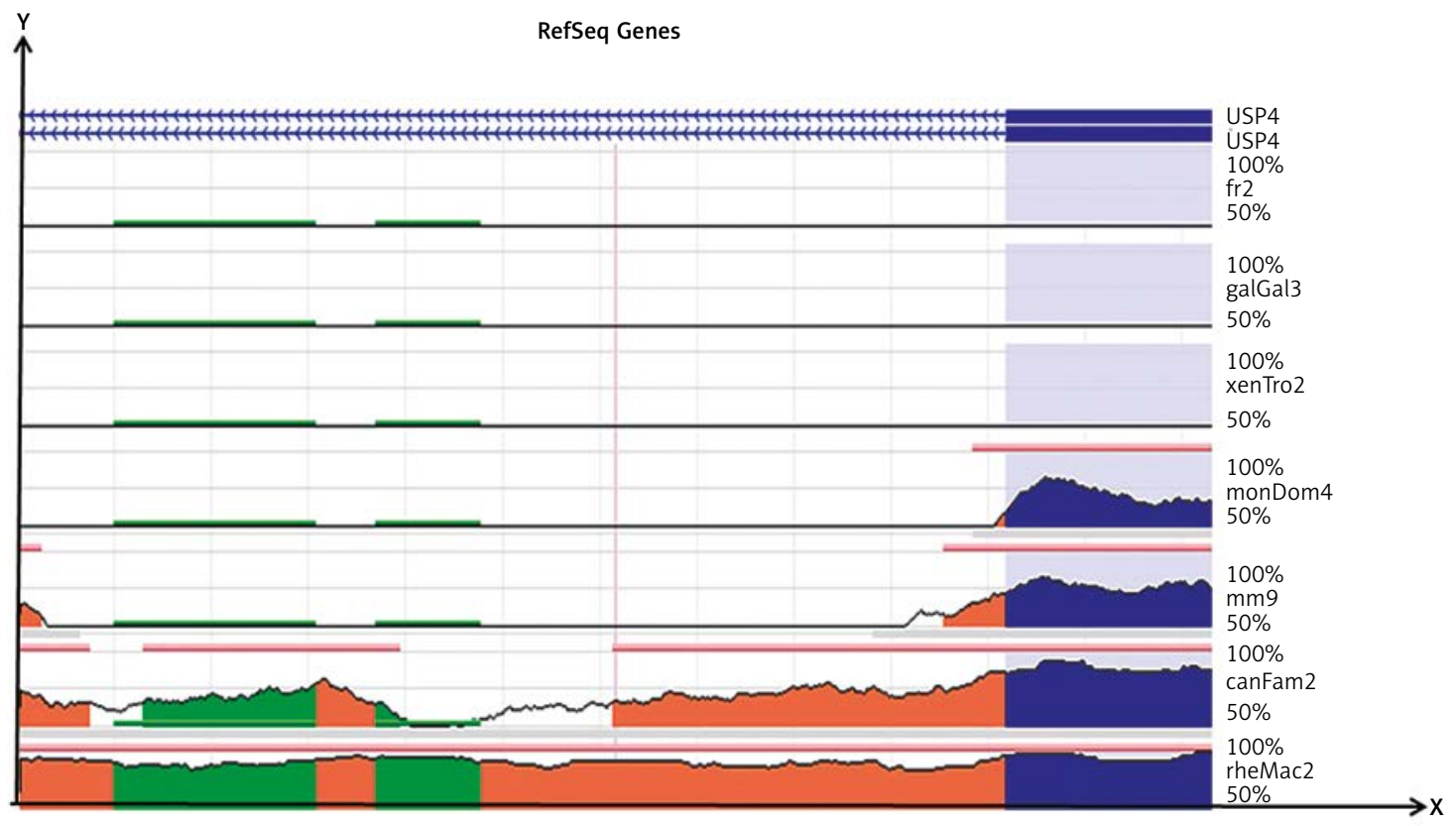

Figure 1. Homology analysis of STGC3 gene gDNA with other vertebrates. The analysis software Evolutionary Conserved Regions (ECRs) was used. The ECR Browser is a dynamic graphical interface that allows users to visualize and analyze ECRs in genomes of sequenced species. The $X$-axis represents positions of bases in the genomes. The $\mathrm{Y}$-axis represents the percentage (\%) identity between the bases of the aligned genomes at a particular position. Usp4, human ubiquitin specific protease 4 encoded by STGC3. Homology analyses were performed for genomics of STGC3 genes between human and other vertebrates. The selected reference genomes are as follows: hg19, human; fr2, Fugu; galGal3, Chicken; xenTro2, Frog; monDom4, Opossum; mm9, Mouse; camFam2, Dog; rheMac2, Rhesus macaque

A

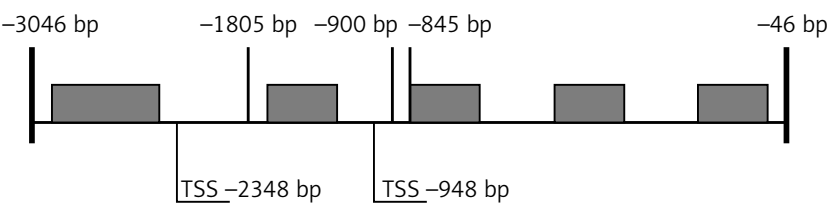

B

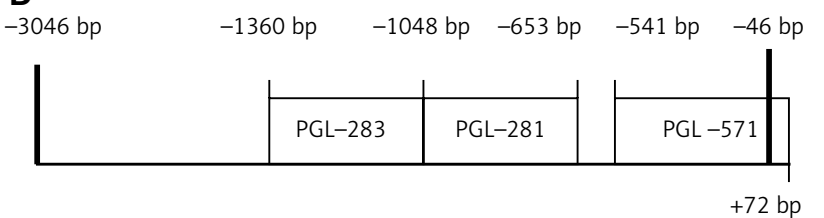

C
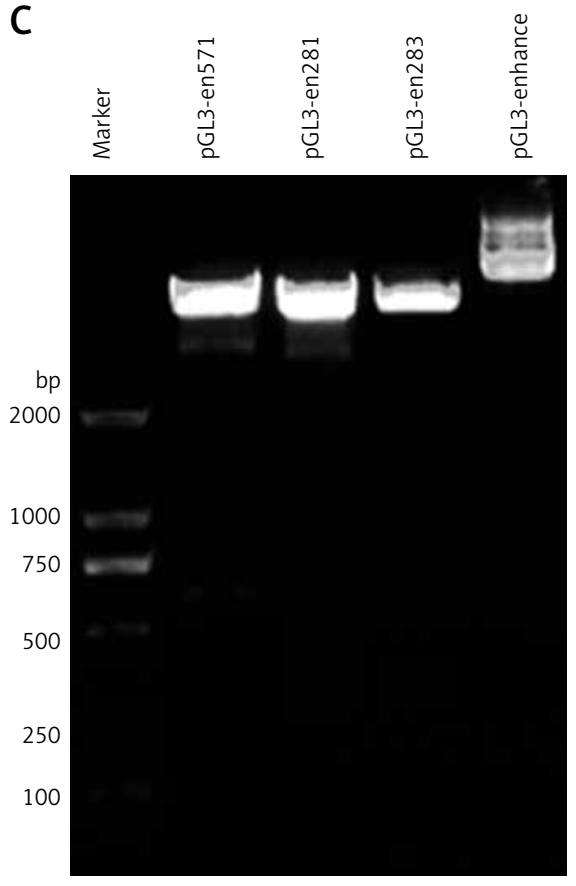

Figure 2. Reporter gene vector construction. A - Diagram of promoter region in STGC3 gene. TSS, gene transcription start site. B - Diagram of vector construction in promoter region of STGC3 gene. $\mathbf{C}$ - Double digested electrophoresis of recombinant plasmid using Mlul and Bglll

Figure 3). It showed the strong promoter activity of plasmid pGL3-en283, pGL3-en281 and pGL3en571 in NP69, 293T and CNE2 cells compared with the negative control pGL3-enhance plasmid. Among them, pGL3-en281 showed the strongest promoter activity, and these three kinds of re- combinant plasmids showed stronger promoter activity in 293T cells than in CNE2 cells. The pGL3en281 plasmid showed stronger promoter activity than PGL3-en571 in the three cells, indicating that $-1048 \mathrm{bp}$ to $-653 \mathrm{bp}$ might be the core promoter region. 
Table I. The RLA results of transfected reporter gene vector in the three cells

\begin{tabular}{|lccc|}
\hline Plasmids & \multicolumn{3}{c|}{ Luciferase relative activity RLA $(\bar{x} \pm \mathbf{s})$} \\
\cline { 2 - 4 } & NP69 cells & 293T cells & CNE2 cells \\
\hline pGL3-control & $0.3920 \pm 0.0201$ & $0.4051 \pm 0.0423$ & $0.3974 \pm 0.0372$ \\
\hline pGL3-en283 & $0.0672 \pm 6.7360 \mathrm{e}-3$ & $0.0600 \pm 0.0294$ & $0.0372 \pm 3.4560 \mathrm{e}-3$ \\
\hline pGL3-en281 & $0.0894 \pm 0.0172$ & $0.2340 \pm 0.0301$ & $0.1090 \pm 4.7870 \mathrm{e}-3$ \\
\hline pGL3-en571 & $0.0760 \pm 0.0101$ & $0.0977 \pm 0.0333$ & $0.0688 \pm 0.0413$ \\
\hline pGL3-enhance & $1.3510 \mathrm{e}-3 \pm 6.3200 \mathrm{e}-5$ & $9.6370 \mathrm{e}-3 \pm 5.4410 \mathrm{e}-3$ & $5.1470 \mathrm{e}-3 \pm 1.8100 \mathrm{e}-4$ \\
\hline
\end{tabular}

\section{Discussion}

Regulation of gene expression is often related to cancers [19-21]. The nasopharyngeal carcinoma associated gene and its expression and regulation mechanisms are important to elucidate the pathogenesis, diagnosis, and treatment of nasopharyngeal carcinoma [2, 3]. As a candidate tumor suppressor gene, early studies showed that STGC3 inhibited the growth and proliferation of tumor cells, and it is found that the LG domain may be essential for the tumor suppression of the STGC3 gene [7-15].

Based on the results of bioinformatics and characteristics of the promoter region, primer design and reporter vector construction of the STGC3 gene were performed. The target fragment included the -1360 bp -1048 bp region containing a promoter fragment with a prediction score of 0.91 and GC boxes. The -1048 bp -653 bp region contained a promoter fragment with a prediction score of 1.0, a transcription initiation site, CpG islands, and a GC box, where there may be the core area of the STGC3 gene promoter. The $-541 \mathrm{bp}$ -+72 bp region contained a promoter fragment with a prediction score of 0.97 and a CAAT box. The reporter gene vector analysis system is the most commonly used tool to study the regulation of gene expression. When a recombinant plasmid transiently transfected cells, identification reports showed the role that the inserted DNA fragment plays in the regulation of gene expression by detecting reporter gene expression levels $[22,23]$. The constructed reporter gene vector transfected cell was detected and calculated for RLA in each cell, which showed the strong promoter activity of pGL3-en283, pGL3-en281 and pGL3-en571 plasmid in NP69, 293T and CNE2 cells compared with negative control plasmid pGL3-enhance. Among them, plasmid pGL3-en281 showed the strongest promoter activity in all three cells, indicating that the $-934 \mathrm{bp}$ to $-653 \mathrm{bp}$ region may be the core promoter region. The STGC3 gene promoter is a TATA-less promoter, which is located in the -2992 bp to -69 bp region. The best promoter region is located $500 \mathrm{bp}$ upstream and $300 \mathrm{bp}$ downstream of the transcription initiation site,

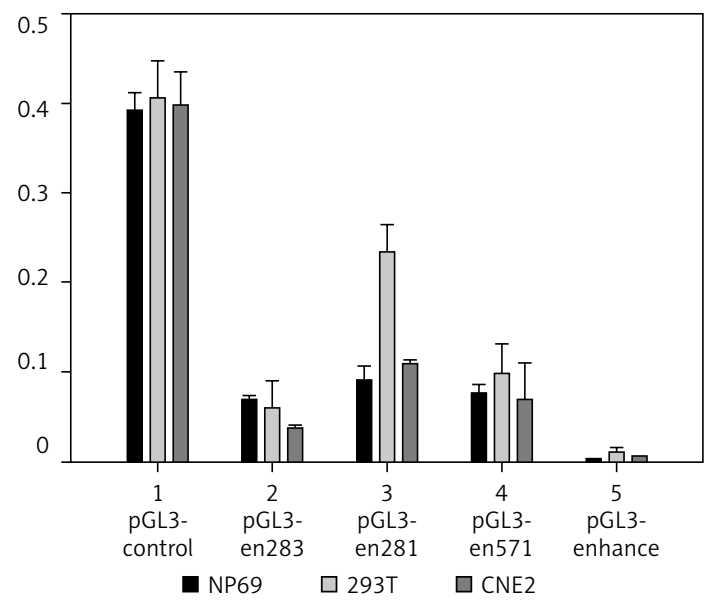

Figure 3. Results of luciferase relative activity of the three reporter gene transfected vectors. pGL3 recombinant plasmids and phRL-SV40 were transfected using Lipofectamine 2000 liposomes. Luciferase reporter gene activity was detected following dual luciferase assay kit steps. Twenty microliters of cell lysate was added to $100 \mu$ l of fluorescence luciferase substrate (LAR II), and liquid scintillation luciferase was measured for the luminescence value after mixing. Then $100 \mu$ l of reaction terminated liquid (Stop \& Glo Reagent) was added and mixed for measurement of internal standard Renilla luciferase with liquid scintillation counter. The ratio of the two is the relative luciferase activity (RLA). The experiments were repeated at least three times

where the CAAT box and GC box are located [2428]. While promoter activity was strongest in the -34 bp to -653 bp region, promoter activity was relatively weak in the -1360 to -1077 and -500 bp to +72 bp region. The promoter activity of pGL3en281 plasmid was stronger than the pGL3-en571 plasmid, indicating a possible negative regulatory element in the -653 bp to +72 bp DNA sequence. These three plasmids showed stronger promoter activity in 293T cells than in CNE2 cells, indicating that factors may interfere with promoter activity in CNE2 cells.

\section{Acknowledgments}

This work was supported by China National Natural Science Foundation (Grant No. 2011GZK46) 
and Hunan Provincial Science and Technology Fund Project (Project No. 2012TT2020).

\section{Conflict of interest}

The authors declare no conflict of interest.

\section{References}

1. Yu WM, Hussain SS. Incidence of nasopharyngeal carcinoma in Chinese immigrants, compared with Chinese in China and South East Asia: review. J Laryngol Otol 2009, 123: 1067-74.

2. Caponiqro F, Lonqo F, Lonna F, et al. Treatment approaches to nasopharyngeal carcinoma: a review. Anticancer Drugs 2010; 21: 471-7.

3. Adham M, Kurniawan AN, Muhtadi AL, et al. Nasopharyngeal carcinoma in Indonesia: epidemiology, incidence, signs, and symptoms at presentation. Chin J Cancer 2012; 31: 185-96.

4. Zhang W, Zeng Z, Zhou Y, et al. Identification of aberrant cell cycle regulation in Epstein-Barr virus-associated nasopharyngeal carcinoma by CDNA microarray and gene set enrichment analysis. Acta Biochim Biophys Sin (Shanghai) 2009; 41: 414-28.

5. Chen J, Hu CF, Hou JH, et al. Epstein-Barr virus encoded latent membrane protein 1 regulates mTOR signaling pathway genes which predict poor prognosis of nasopharyngeal carcinoma. J Transl Med 2010; 8: 30.

6. Liu Y, Fang Z, Liu L, et al. Detection of Epstein-Barr virus DNA in serum or plasma for nasopharyngeal cancer: a meta-analysis. Genet Test Mol Biomarkers 2011; 15 : 495-502.

7. Amato E, Barbi S, Malpeli G, et al. Chromosome 3p alterations in pancreatic endocrine neoplasia. Virchows Arch 2011; 458: 39-45.

8. He XS, Xiao ZQ, Chen ZC, et al. The new STGC3 gene cloning and preliminary functional analysis. Chin J Cancer 2004; 23: 1110-5.

9. He XS, Chen ZC, Tian F, et al. Identification of nasopharyngeal chromosome 3 p21 region in a down-regulation EST. Chin J Cancer 2003; 22: 1-5.

10. Deng M, He XS, Luo Q, et al. Tet Regulates STGC3 gene expression in CNE2 cell line. Prog Biochem Biophys 2006; 33: 39-44.

11. Qiu QC, Hu B, He XS, et al. Experimental studies of Tet regulation and control of the CNE2 cell lines with STGC3 over-expression in nude mice tumor. Prog Biochem Biophys 2007; 34: 359-365.

12. He XS, Deng M, Yang S, et al. The tumor supressor function of STGC3 and its reduced expression in nasopharyngeal carcinoma. Cell Mol Biol Lett 2008; 13: 339-52.

13. Hu B, Qiu QC, He XS, et al. Effects of estrogen on growth and proliferation of CNE2 cell lines with STGC3 overexpression. Biochem Biophys 2007; 34: 538-45.

14. Rong YH, He XS, Li YL, et al. Effects of up-regulated STGC3 expression on protein expression profile in CNE2 cell lines. J Nanhua Univ 2005; 33: 171-5.

15. Li L, He XS, Luo Q, et al. Effects of STGC3 gene deletion on growth and proliferation of CNE2 cells. Biochem Biophys 2011; 38: 248-53.

16. The ENCODE Project Consortium. Identification and analysis of functional elements in $1 \%$ of the human genome by the ENCODE pilot project. Nature 2007; 447: 799-816.

17. Denoeud F, Kapranov P, Ucla C, et al. Prominent use of distal 5 'transcription start sites and discovery of a large number of additional exons in ENCODE regions. Genome Res 2007; 17: 746-59.

18. Wu JQ, Du J, Rozowsky J, et al. Systematic analysis of transcribed loci in ENCODE regions using RACE sequencing reveals extensive transcription in the human genome. Genome Biol 2008; 9: R3.

19. Jabłonowski Z, Reszka E, Gromadzińska J, et al. Hypermethylation of $p 16$ and DAPK promoter gene regions in patients with non-invasive urinary bladder cancer. Arch Med Sci 2011; 7: 512-6.

20. Liang QL, Wang BR, Li ZY, et al. Construction of eukaryotic expression vector of TSLC1 gene. Arch Med Sci 2011; 7: 579-85.

21. El-Din HG, Ghafar NA, Saad NE, et al. Relationship between codon 249 mutation in exon 7 of p53 gene and diagnosis of hepatocellular carcinoma. Arch Med Sci 2010; 6: 348-55.

22. McNabb DS, Reed R, Marciniak RA. Dual luciferase assay system for rapid assessment of gene expression in Saccharomyces cerevisiae. Eukaryot Cell 2005; 4: 1539-49.

23. Alcaraz-Perez F, Mulero V, Cayuela ML. Application of the dual-luciferase reporter assay to the analysis of promoter activity in Zebrafish embryos. BMC Biotechnol 2008; 8: 81.

24. Ovcharenko I, Nobrega MA, Loots GG, et al. ECR Browser: a tool for visualizing and accessing data from comparisons of multiple vertebrate genomes. Nucleic Acids Research 2004; 32: W280-6.

25. Wolner BS, Gralla JD. Roles for non-TATA core Promoter sequences in transcription and factor binding. Mol Cell Biol 2000; 20: 3608-15.

26. Anish R, Hossain MB, Jacobson RH, et al. Characterization of transcription from TATA-less promoters: identification of a new core promoter element XCPE2 and analysis of factor requirements. PloS one 2009; 4: e5130.

27. Zhong P, Melcher K. Identification and characterization of the activation domain of Ifh1, an activator of model TATA-less genes. Biochem Biophys Res Commun 2010; 392: 77-82

28. Down TA, Hubbard TJ. Computational detection and location of transcription Start sites in mammalian genomic DNA. Genome Res 2002; 12: 458-61. 